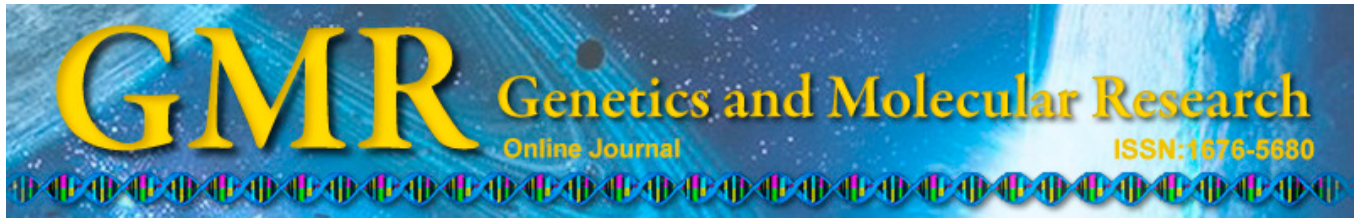

\title{
Diagnostic performance of microRNA-29a for colorectal cancer: a meta-analysis
}

\author{
M.L. Zhi ${ }^{1 *}$, Z.J. Liu ${ }^{2 *}$, X.Y. Yi ${ }^{1}$, L.J. Zhang ${ }^{1}$ and Y.X. Bao ${ }^{1}$ \\ ${ }^{1}$ Department of Clinical Laboratory, \\ The Second Affiliated Hospital of Chongqing Medical University, \\ Chongqing, China \\ ${ }^{2}$ Department of Clinical Medicine, Xinjiang Medical University, \\ Xinjiang, China \\ *These authors contributed equally to this study. \\ Corresponding author: Y.X. Bao \\ E-mail: yixibao020278@sina.com
}

Genet. Mol. Res. 14 (4): 18018-18025 (2015)

Received June 18, 2015

Accepted September 8, 2015

Published December 22, 2015

DOI http://dx.doi.org/10.4238/2015.December.22.28

\begin{abstract}
Previous studies have revealed that the expression level of microRNA-29a (miR-29a) was remarkably different in colorectal cancer (CRC) patients and healthy controls, indicating that miR-29a can be used as a diagnostic marker of $\mathrm{CRC}$, but the results have been inconsistent. We conducted this meta-analysis to assess the diagnostic performance of blood-based miR-29a for CRC. We performed a systematic review of studies published over the past two decades to investigate the diagnostic performance of serum miR-29a for the diagnosis of CRC. QUADAS-2 was used to evaluate the quality of the studies. Performance characteristics (diagnostic sensitivity, specificity, and other measures of accuracy) were pooled and examined using random-effect models. Five studies, which included 281 CRC patients and 299 healthy controls, met the inclusion criteria. The summary estimates for miR-29a in CRC diagnoses showed a diagnostic sensitivity of $0.59(95 \% \mathrm{CI}=0.53-0.65)$,
\end{abstract}


a specificity of $0.89(95 \% \mathrm{CI}=0.85-0.93)$, and a diagnostic odds ratio of $12.22(95 \% \mathrm{CI}=5.07-29.44)$. The area under curve and Q value for the summary receiver operating characteristic curves were 0.9128 and 0.8453 , respectively. In conclusion, miR-29a may be a novel potential biomarker for CRC diagnosis.

Key words: Biomarker; Colorectal cancer; Meta-analysis; MicroRNA-29a; Diagnostic marker

\section{INTRODUCTION}

Colorectal cancer (CRC) is one of the most prevalent malignancies. In 2008, there were 1.2 million new cases of CRC and 608,700 deaths related to this disease, making CRC the second most common cancer in females and the third most common in males (Jemal et al., 2011). Numerous studies have shown that early diagnosis is a key factor in fighting this disease. It has been suggested that $>95 \%$ of patients with CRC would benefit from curative surgery if diagnoses were made at an early stage (Bond, 2000; Pawa et al., 2011). To date, several CRC screening strategies have been implemented to detect CRC. Colonoscopy has been used to diagnose CRC for many years; however, because of its invasive character, it can be painful and often unacceptable to a significant percentage of the at-risk population. A previous study demonstrated that the compliance rate of recommended colonoscopy for the population aged from 50-75 years is only 50-75\% in the US (Fesler et al., 2014). The fecal occult blood test is another traditional screening test for CRC and is safe and convenient, but its sensitivity is low. These methods are also compromised by either low cost-effectiveness or limited diagnostic accuracy. As a result, there is a need for non-invasive, cost-effective, and accurate biomarkers.

MicroRNAs are small non-coding RNA molecules of 18-22 nucleotides in length, which can regulate the expression of target genes at the post-transcriptional level by binding to 3'-untranslated region of target messenger RNAs to control some cellular processes in eukaryotic organisms (Krol et al., 2010). MicroRNAs can act as tumor suppressor genes or oncogenes to influence the growth and metastasis of tumors, suggesting that microRNAs may play an important role in human cancer (Fabbri et al., 2008). In recent years, a series of emerging studies have shown that microRNAs may be associated with many types of cancer (Mazeh et al., 2013; Guo et al., 2014; Morishita and Masaki, 2015). Compared with other clinical biomarkers, microRNAs are extremely stable, the expression level of microRNAs is tissue-specific, and expression is changed in parallel with diverse biological stages (Hanson et al., 2009; Etheridge et al., 2011). In addition, microRNAs can be measured by microarrays and quantitative polymerase chain reaction-based methods, and extracellular microRNAs can be found in plasma and other body fluids (Weber et al., 2010). Thus, blood-based microRNAs may be useful as biomarkers for cancer.

MicroRNA-29a (miR-29a) is a cancer-related microRNA first reported by LagosQuintana et al. (2001) in HeLa cells. In recent years, various studies have shown that miR-29a expression was related to many solid cancers. Over the past few years, additional studies have revealed that miR-29a is significantly up-regulated in the serum of CRC patients compared to that in healthy individuals (Huang et al., 2010; Feng et al., 2013; Brunet Vega et al., 2013; Giráldez et al., 2013; Luo et al., 2013). These studies have demonstrated the diagnostic value of miR-29a for CRC; however, the pooled sensitivity and specificity were not evaluated and 
the quality of the included studies was not evaluated. To investigate the overall diagnostic performance of serum miR-29a for CRC, we performed a meta-analysis.

\section{MATERIAL AND METHODS}

\section{Selection of studies}

EMBASE, PubMed, Google Scholar, Chinese National Knowledge Infrastructure, and Wan Fang Database databases were searched to identify eligible studies published before July 2014. A manual search was also performed using the references listed in the original and review articles, which were found by searching the databases. The key words for the literature search included: mir29a; microRNA29a; colorectal carcinoma; CRC; and diagnosis. No language restrictions were applied.

\section{Inclusion and exclusion criteria}

Studies were included if they met all of the following criteria: 1) CRC diagnosis was based on colonoscopy or histological examination; 2) the number of patients or controls were must more than 20;3) only individuals with a negative result of colonoscopy and without a history of any types of cancer were recruited as controls; 4) the performance of miR-29a was assessed alone; 5) blood was the only sample type and was collected prior to any treatment; 6) presence of data on sensitivity and specificity, or such values could be obtained from the raw data. The exclusion criteria were as follows: 1) studies conducted on samples other than human serum; 2) duplicate publications; 3) reviews, editorials, letters, case reports, and conference abstracts.

\section{Data extraction}

Data extraction was independently conducted by 2 reviewers (M.L. Zhi and X.Y. Yi). The following data were extracted: name of the first author, year of publication, numbers of patients and controls, TNM stage, journal, distribution of age, cut-off values, and data for $2 \mathrm{x}$ 2 table. Disagreements were resolved through discussion to reach a consensus.

\section{Quality assessment}

The quality of each article was evaluated independently by 2 investigators (Z.J. Liu and L.J. Zhang) based on the Quality Assessment of Diagnostic Accuracy Studies 2 (QUADAS-2). The QUADAS-2 is a reformative tool that includes 4 key domains (patient selection, index test, reference standard, flow and timing) supported by signaling questions to aid in judgment of the risk of bias, rating risk of bias, and concerns regarding applicability as "high", "unclear", and "low" (Whiting et al., 2011).

\section{Statistical analysis}

All analyses of relevant data were accomplished using the Meta-DiSc statistical software. Spearman correlation analysis evaluates heterogeneity caused by the threshold effect. 
Heterogeneity resulting from the non-threshold effect was assessed by the chi-square method and the I-square $\left(\mathrm{I}^{2}\right)$ test. Values of $\mathrm{P} \leq 0.05$ were considered to be statistically significant. True positives, false positives, true negatives, and false negatives were extracted from each study, and these data were used to obtain pooled sensitivity, specificity, positive likelihood ratio, negative likelihood ratio, diagnostic odds ratio (DOR), and their $95 \%$ confidence interval $(95 \% \mathrm{CI})$, as well as the summary receiver operator characteristic curve (SROC), and the area under the curve.

\section{RESULTS}

\section{Study characteristics}

After a series of related searches, a total of 76 studies were identified, 43 of which remained after duplicates were removed. Based on the titles and abstracts, 23 reviews were removed. A total of 20 articles were considered potentially applicable for our meta-analysis, and the full texts of these articles were obtained. The articles were read and 15 articles were excluded for the following reasons: 4 studies did not conduct diagnosis, 3 did not use bloodbased samples, 3 were not related to CRC, 4 did not examine miR-29a, and 1 did not include data. Finally, 5 studies met our inclusion and exclusion criteria (Figure 1).

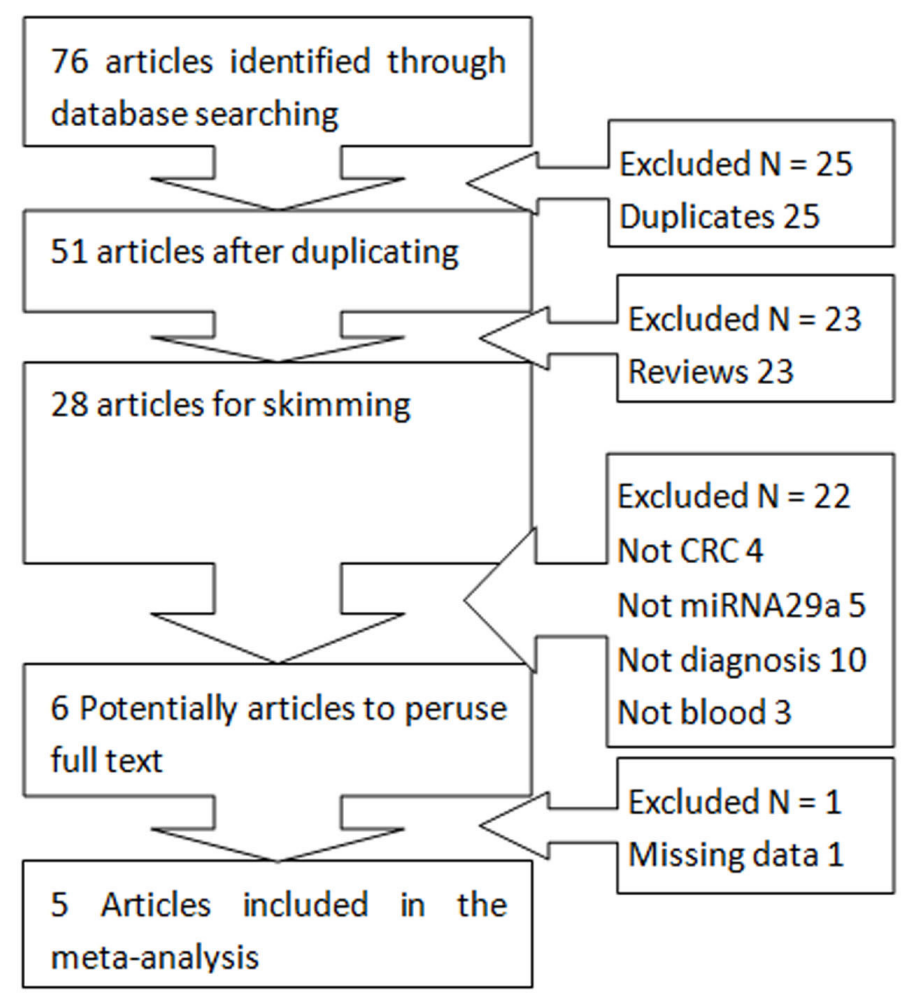

Figure 1. Flow chart showing study selection procedure. 
In the present meta-analysis, one of the eligible studies was published in 2010 and the remaining studies were published in 2013. The overall sample size in this study was 580 (281 patients with CRC and 299 healthy controls). The main characteristics of the studies, such as the first author, publication year, numbers of patients and controls, TNM stage, true positives, false positives, true negatives, and false negative are outlined in Table 1.

\begin{tabular}{|c|c|c|c|c|c|c|c|}
\hline Author (year) & $\mathrm{CRC} /$ controls & TNM (I/II/III/IV) & Cut-off values & True positive & False positive & False negative & True negative \\
\hline Huang (2010) & $100 / 59$ & $27 / 25 / 38 / 10$ & 1.33 & 69 & 6 & 31 & 53 \\
\hline Brunet Vega (2013) & 3) $30 / 26$ & $0 / 0 / 36 / 0$ & Unknown & 23 & 1 & 7 & 25 \\
\hline Giraldez (2013) & $21 / 20$ & $4 / 8 / 6 / 3$ & 0.545 & 13 & 3 & 8 & 17 \\
\hline Luo (2013) & $80 / 144$ & $22 / 25 / 26 / 5 / 2^{*}$ & 7.2 & 24 & 14 & 56 & 130 \\
\hline Feng et al. (2013) & $50 / 50$ & Unknown & Unknown & 36 & 8 & 14 & 42 \\
\hline
\end{tabular}

*Patients were not reported.

\section{Quality assessment}

The quality of each study was assessed using the QUADAS-2 software. The "risk of bias" and "applicability concerns" were summarized by judging each domain for each study included. In this meta-analysis, all 5 articles were considered to be of upper quality (Figure 2).

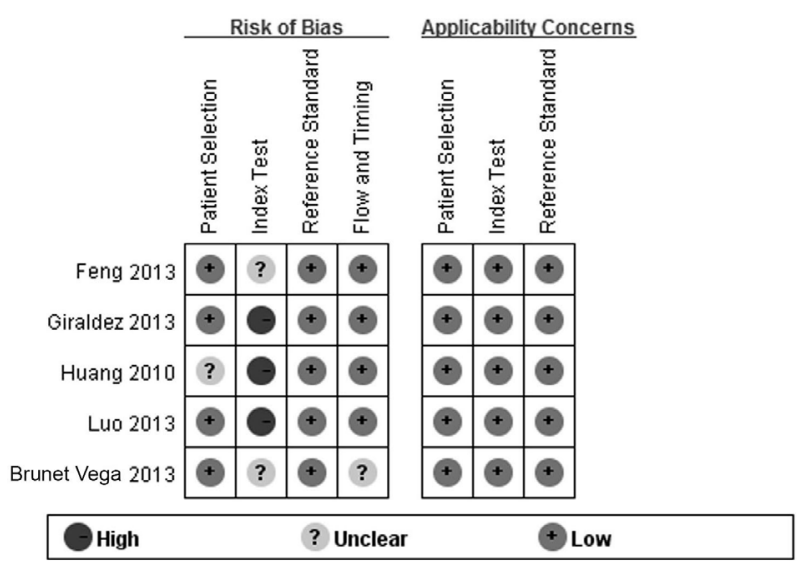

Figure 2. Quality assessment of studies included (QUADAS-2).

\section{Data analysis}

Within the studies selected, heterogeneity was induced by the non-threshold effect, and thus the random-effect model was used to estimate overall value of miR-29a for diagnosing CRC. For miR-29a, the sensitivity (Figure 3), specificity (Figure 4), and DOR (Figure 5) of the 5 articles included are shown as forest plots. A pooled sensitivity and specificity of miR29 a were $59(95 \% \mathrm{CI}=0.53-0.65)$ and $89(95 \% \mathrm{CI}=0.85-0.93)$. Its positive likelihood ratio and negative likelihood ratio in diagnosis of $\mathrm{CRC}$ were $4.66(95 \% \mathrm{CI}=3.01-7.22)$ and 0.41 $(95 \% \mathrm{CI}=0.24-0.70)$, respectively. The diagnostic odds ratio and the area under SROC were $12.22(95 \% \mathrm{CI}=5.07-29.44)$ and $91.28 \%$, respectively (Figure 6). 


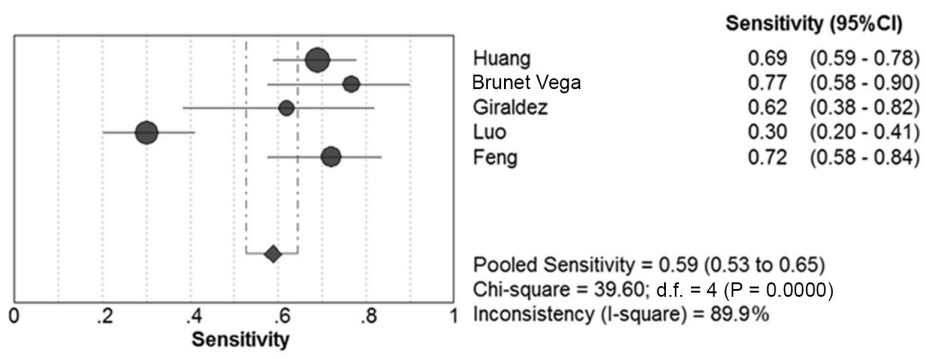

Figure 3. Forest plot of sensitivity for miR-29a.

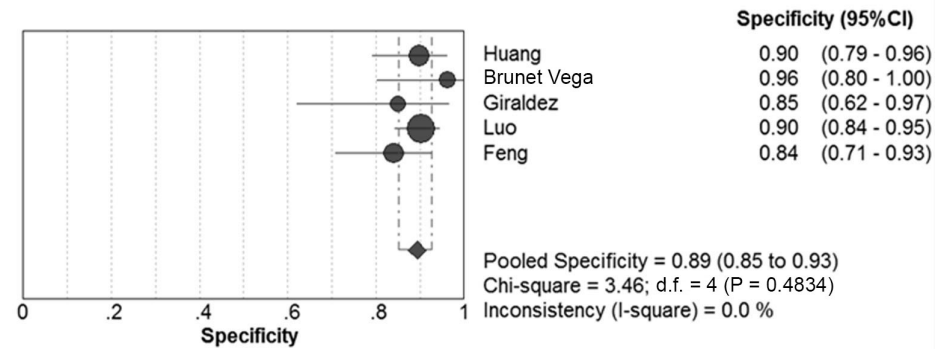

Figure 4. Forest plot of specificity of miR-29a.

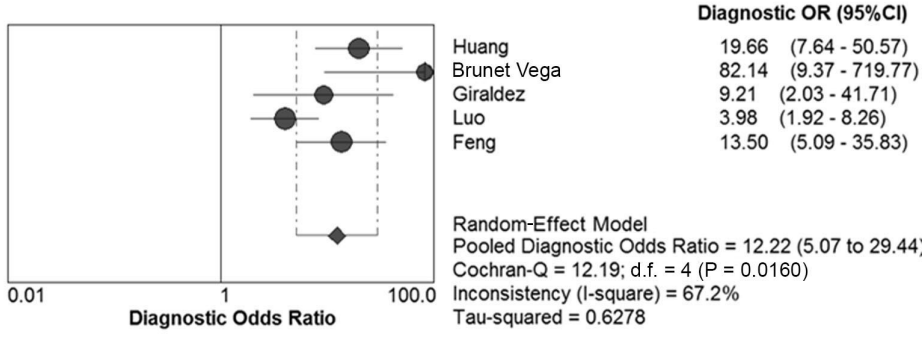

Figure 5. Forest plot of diagnostic odds ratio of miR-29a.

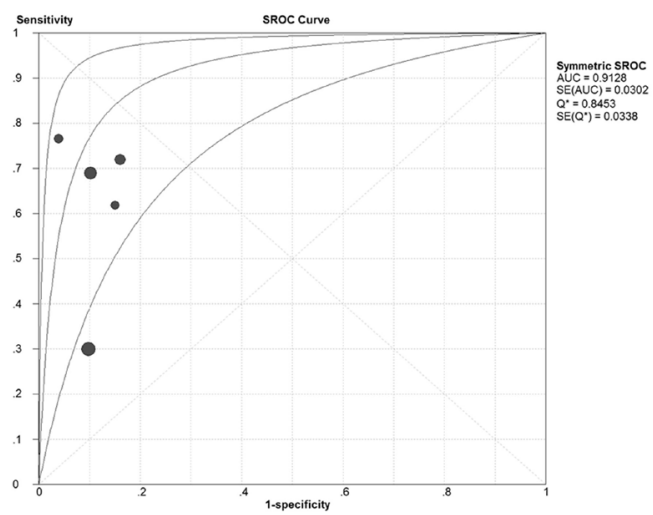

Figure 6. MicroRNA-29a of summary receiver operator characteristic curves. 


\section{DISCUSSION}

In this meta-analysis, the pooled specificity and positive likelihood ratio of serum miR-29a were 0.89 and 4.66, respectively, indicating that patients with CRC have an approximately 5 -fold higher chance of being miR-29a-positive compared to those without CRC. DOR is an overall indicator of diagnostic test performance, and the value of DOR ranges from 0 to infinity, with higher values representing better test performance (Glas et al., 2003). For example, a value of $\mathrm{DOR}=1.0$ suggests that the test could not distinguish between patients and controls. Our meta-analysis showed that the overall DOR of miR-29a for CRC diagnosis was 12.22 , indicating that the probability of miR-29a-positive CRC patients was approximately 13-fold higher than in normal controls. The SROC curve, which combines data from all relevant studies, represents the trade-off between sensitivity and specificity. The area under the curve of SROC curve is an overall summary of test performance; the area under the curve in this study was 0.91 , demonstrating that miR-29a could be used to accurately diagnose CRC (Jones and Athanasiou, 2005). The Q value is another important indicator used to assess the diagnostic accuracy of the index test. The $\mathrm{Q}$ value in this meta-analysis was 0.85 , indicating that both diagnostic sensitivity and specificity would achieve 0.85 by adjusting the diagnostic threshold. Otherwise, the pooled sensitivity of serum miR-29a was 0.59 , which may be insufficient for ruling out CRC. These results suggest that miR-29a may be a novel diagnostic biomarker for CRC.

miR-29a is a promising marker for diagnosing CRC. First, miR-29a was characterized by high stability, and Mitchell et al. (2008) suggested that microRNAs are present in human blood in a remarkably stable form that is protected from endogenous RNase activity. Second, miR-29a is present in plasma and other body fluids and can be measured easily. Finally, miR29a has higher sensitivity compared to some traditional biomarkers. For example, carcinoembryonic antigen is a common marker for CRC diagnosis. However, the overall sensitivity of carcinoembryonic antigen for diagnosis CRC varied between 43 and $69 \%$ in a previous study (Hundt et al., 2007).

There were several limitations in our study. First, miR-29a is a blood biomarker for CRC diagnosis but was only discovered recently; therefore, the total sample size in our study was small. In contrast, all studies recruited healthy individuals as controls, which may weaken the diagnostic accuracy.

In conclusion, the present meta-analysis showed that miR-29a may be a promising biomarker for diagnosing CRC. Additional multi-center studies with larger sample sizes should be performed.

\section{Conflicts of interest}

The authors declare no conflict of interest.

\section{ACKNOWLEDGMENTS}

Research supported by funds of the National Natural Science Foundation of China (\#81274144 and \#81473388). 


\section{REFERENCES}

Bond JH (2000). Clinical evidence for the adenoma-carcinoma sequence, and the management of patients with colorectal adenomas. Semin. Gastrointest. Dis. 11: 176-184.

Brunet Vega A, Pericay C, Moya I, Ferrer A, et al. (2013). MicroRNA expression profile in stage III colorectal cancer: circulating miR-18a and miR-29a as promising biomarkers. Oncol. Rep. 30: 320-326.

Etheridge A, Lee I, Hood L, Galas D, et al. (2011). Extracellular microRNA: a new source of biomarkers. Mutat. Res. 717: 85-90.

Fabbri M, Croce CM and Calin GA (2008). MicroRNAs. Cancer J. 14: 1-6.

Feng L, Pang Z,Shang S, Wu B, et al. (2013). Circulating miR-29a and miR-92a in serum: Evidence against their as biom arkers in colorectal cancer. Chin. J. Clin. Oncol. Rehab. 12: 19-24.

Fesler A, Jiang J, Zhai H and Ju J (2014). Circulating microRNA testing for the early diagnosis and follow-up of colorectal cancer patients. Mol. Diagn. Ther. 18: 303-308.

Giráldez MD, Lozano JJ, Ramírez G, Hijona E, et al. (2013). Circulating microRNAs as biomarkers of colorectal cancer: Results from a genome-wide profiling and validation study. Clin. Gastroenterol. Hepatol. 11: 681-688.

Glas AS, Lijmer JG, Prins MH, Bonsel GJ, et al. (2003). The diagnostic odds ratio: a single indicator of test performance. J. Clin. Epidemiol. 56: 1129-1135.

Guo Z, Zhao C and Wang Z (2014). MicroRNAs as ideal biomarkers for the diagnosis of lung cancer. Tumour Biol. 35: 10395-10407.

Hanson EK, Lubenow H and Ballantyne J (2009). Identification of forensically relevant body fluids using a panel of differentially expressed microRNAs. Anal. Biochem. 387: 303-314.

Huang Z, Huang D, Ni S, Peng Z, et al. (2010). Plasma microRNAs are promising novel biomarkers for early detection of colorectal cancer. Int. J. Cancer 127: 118-126.

Hundt S, Haug U and Brenner H (2007). Blood markers for early detection of colorectal cancer: a systematic review. Cancer Epidemiol. Biomarkers Prev. 16: 1935-1953.

Jemal A, Bray F, Center MM, Ferlay J, et al. (2011). Global cancer statistics. CA Cancer J. Clin. 61: 69-90.

Jones CM and Athanasiou T (2005). Summary receiver operating characteristic curve analysis techniques in the evaluation of diagnostic tests. Ann. Thorac. Surg. 79: 16-20.

Krol J, Loedige I and Filipowicz W (2010). The widespread regulation of microRNA biogenesis, function and decay. Nat. Rev. Genet. 11: 597-610.

Lagos-Quintana M, Rauhut R, Lendeckel W and Tuschl T (2001). Identification of novel genes coding for small expressed RNAs. Science 294: 853-858.

Luo X, Stock C, Burwinkel B and Brenner H (2013). Identification and evaluation of plasma microRNAs for early detection of colorectal cancer. PLoS One 8: e62880.

Mazeh H, Mizrahi I, Ilyayev N, Halle D, et al. (2013). The diagnostic and prognostic role of microRNA in colorectal cancer - a comprehensive review. J. Cancer 4: 281-295.

Mitchell PS, Parkin RK, Kroh EM, Fritz BR, et al. (2008). Circulating microRNAs as stable blood-based markers for cancer detection. Proc. Natl. Acad. Sci. 105: 10513-10518.

Morishita A and Masaki T (2015). MicroRNAs in hepatocellular carcinoma. Hepatol. Res. 45: 128-141.

Pawa N, Arulampalam T and Norton JD (2011). Screening for colorectal cancer: established and emerging modalities. Nat. Rev. Gastroenterol. Hepatol. 8: 711-722.

Weber JA, Baxter DH, Zhang S, Huang DY, et al. (2010). The microRNA spectrum in 12 body fluids. Clin. Chem. 56: 1733-1741.

Whiting PF, Rutjes AW, Westwood ME, Mallett S, et al. (2011). QUADAS-2: a revised tool for the quality assessment of diagnostic accuracy studies. Ann. Intern. Med. 155: 529-536. 